Revue internationale P.M.E.

Économie et gestion de la petite et moyenne entreprise

\title{
PMI exportatrices et transformations de la stratégie dans les pays en transition
}

\section{Stéphan Bourcieu}

Volume 14, numéro 1, 2001

URI : https://id.erudit.org/iderudit/1008687ar

DOI : https://doi.org/10.7202/1008687ar

Aller au sommaire du numéro

\section{Éditeur(s)}

Presses de l’Université du Québec

ISSN

0776-5436 (imprimé)

1918-9699 (numérique)

Découvrir la revue

Citer cette note

Bourcieu, S. (2001). PMI exportatrices et transformations de la stratégie dans les pays en transition. Revue internationale P.M.E., 14(1), 69-92.

https://doi.org/10.7202/1008687ar

\section{Résumé de l'article}

À la différence d'autres marchés engagés dans le contexte de mondialisation des affaires, l'ouverture des marchés issus de la dissolution de l'Union soviétique offre des options stratégiques considérables pour les PMI exportatrices. La complexité du processus de transition, les imbrications entre les institutions et acteurs économiques locaux, les disparités réglementaires, tout autant que des besoins spécifiques, ouvrent sans cesse de nouveaux horizons. Cette instabilité permet aux PMI d'être compétitives par rapport aux entreprises mondialisées, pourtant attirées très tôt par ce " far east ».

Cet article présente les résultats d'une recherche longitudinale et monographique sur la formation et les mutations de l'offre des PMI dans ce contexte de transition ; elle s'inscrit dans le cadre d'une étude visant à analyser leurs stratégies internationales sur ces marchés.

Notre analyse s’attache à démontrer le caractère incrémental de la stratégie, rendu nécessaire pour réagir aux spécificités de l'environnement institutionnel local. Nous étudierons plus particulièrement comment une PMI, à partir d'une stratégie internationale traditionnelle de spécialisation, peut être amenée à développer une stratégie pour l'ensemble de la filière et ainsi orienter favorablement l'environnement dans lequel elle évolue. Partant de ce cas, cette approche exploratoire tentera de relever les caractéristiques d'une stratégie de filière adaptée aux PMI.
Ce document est protégé par la loi sur le droit d'auteur. L'utilisation des services d'Érudit (y compris la reproduction) est assujettie à sa politique d'utilisation que vous pouvez consulter en ligne.

https://apropos.erudit.org/fr/usagers/politique-dutilisation/ 


\title{
Notes de recherche \\ PMl exportatrices et transformations de la stratégie dans les pays en transition
}

Stéphan BOURCIEU

ISEOR, Université Lumière Lyon II

\author{
MOTS CLÉS
}

\section{PME - Internationalisation - Environnement institutionnel Stratégie de filière}

\begin{abstract}
RÉSUMÉ
À la différence d'autres marchés engagés dans le contexte de mondialisation des affaires, l'ouverture des marchés issus de la dissolution de l'Union soviétique offre des options stratégiques considérables pour les PMI exportatrices. La complexité du processus de transition, les imbrications entre les institutions et acteurs économiques locaux, les disparités réglementaires, tout autant que des besoins spécifiques, ouvrent sans cesse de nouveaux horizons. Cette instabilité permet aux PMI d'être compétitives par rapport aux entreprises mondialisées, pourtant attirées très tôt par $c e$ «far east».

Cet article présente les résultats d'une recherche longitudinale et monographique sur la formation et les mutations de l'offre des PMI dans ce contexte de transition; elle s'inscrit dans le cadre d'une étude visant à analyser leurs stratégies internationales sur ces marchés.
\end{abstract}

\section{L'AUTEUR}

StÉPHAN BOURCIEU est doctorant en sciences de gestion à l'Université Lumière Lyon II (Centre de recherche ISEOR) et bénéficiaire d'une convention CIFRE de recherche en entreprise. Ses recherches portent sur les conditions du développement de l'entrepreneuriat des pays en transition, les coopérations interentreprises et les PME en contexte international. Adresse: ISEOR, 15, chemin du Petit-Bois, 69130 Ecully, France. Courriel : <SBourcieu@aol.com>.

Je tiens à remercier les trois lecteurs anonymes pour la pertinence de leurs remarques et tout particulièrement le premier lecteur. Je remercie également l'Association nationale pour la recherche technologique pour le soutien qu'elle apporte à cette recherche. 
Notre analyse s'attache à démontrer le caractère incrémental de la stratégie, rendu nécessaire pour réagir aux spécificités de l'environnement institutionnel local. Nous étudierons plus particulièrement comment une PMI, à partir d'une stratégie internationale traditionnelle de spécialisation, peut être amenée à développer une stratégie pour l'ensemble de la filière et ainsi orienter favorablement l'environnement dans lequel elle évolue. Partant de ce cas, cette approche exploratoire tentera de relever les caractéristiques d'une stratégie de filière adaptée aux PMI.

\section{ABSTRACT}

Unlike other markets involved in the globalization of business, the opening of the markets which have emerged from the break-up of the Soviet Union, offers considerable strategic opportunities for exporting SMI. The complexities of the process of transition, the relationships between the locally active persons and bodies, the statutory disparities, as well as specific needs, are constantly opening up new horizons. The instability enables the SMIs to be competitive with globalized businesses, although the latter were very soon attracted by the "far east".

The objective of this article is to present the results of a longitudinal and qualitative study of the creation and transformation of the SMIs' strategy in this transitional context. It forms part of a broader study regarding their strategies of internationalization in these markets.

Our analysis aims to show the incremental character of the way in which the strategy is formulated, necessitated by the institutional requirements of the local environment. We shall study in particular how SMIs, within a strategy of specialization, can be led to develop their offer over the whole sector of activity, and how they can thus favourably direct the development of the environment in which they are active. Finally, based on the exploratory approach, we shall attempt to define the main characteristics of a "strategy of filière" adapted for SMIs.

\section{RESUMEN}

A diferencia de otros mercados involucrados en el contexto de la mundialización de los negocios, la apertura de los mercados nacidos de la descomposición de la Unión Soviética ofrece oportunidades estratégicas considerables para las pequeñas y medianas industrias exportadoras. La complejidad del proceso de transición, los entrelazamientos entre las instituciones y los actores económicos locales, las disparidades reglamentarias, al igual que las necesidades específicas, abren sin cesar nuevos horizontes. Esta inestabilidad permite a las pequeñas y medianas industrias ser competitivas frente a empresas mundializadas, atraídas sin embargo muy temprano por este "Lejano Este »

Este artículo presenta los resultados de una investigación longitudinal y monográfica sobre la formación y las mutaciones de la oferta de las pequeñas y medianas industrias en este marco de transición. Se inscribe en el contexto de un estudio en relación con el análisis de sus estrategias internacionales sobre estos mercados. 
Nuestro análisis intenta demostrar el caracter incremental de la estrategia, necesaria para responder a las especificidades del entorno institucional local. Estudiaremos más en particular cómo una pequeña y mediana industria, con una estrategia internacional tradicional de especialización puede ser llevada a desarrollar su estrategia sobre el conjunto de la rama y de esta forma orientar favorablemente el entorno en el cual evoluciona. En este caso, intentaremos definir los contornos de una estratregia de rama adaptada a las pequeñas y medianas industrias.

\section{ZUSAMMENFASSUNG}

Im Unterschied zu anderen der Globalisierung unterworfenen Märkten, stellt die Aufsplittung der Sowjetunion beträchtliche strategische Optionen dar, für exportierende kleine und mittlere Industrieunternehmen. Die Komplexität des Übergangsprozesses, die Verflechtung zwischen den Institutionen und den regionalen, wirtschaftlichen Akteuren, die reglementarischen Ungleichheiten und die spezifischen Bedürfnisse, ermöglichen, ohne Unterbruch neue Horizonte zu erschliessen. Diese Instabilität erlaubt den kleinen und mittleren Industrieunternehmen wettbewerbsfähig zu sein gegenüber den global tätigen Unternehmen, die sich sehr früh auf diesen 'far east' orientiert haben.

Dieser Artikel zeigt die Ergebnisse einer Langzeitstudie über die Bildung und Veränderung des Angebotes von kleinen und mittleren Industrieunternehmen im erwähnten Übergangsprozess.

Unsere Analyse versucht aufzuzeigen, dass die Strategie der Unternehmen in kleinen Schritten an die spezifischen lokalen Bedingungen angepasst wurde. Im speziellen Fall untersuchen wir, wie ein kleines und mittleres Industrieunternehmen ihre auf Spezialisierung bedachte internationale Strategie auf alle Filialen entwickelt hat, um den spezifischen regionalen Gegebenheiten besser gerecht zu werden. Ausgehend von diesem Fall, beziehungsweise dieser Annäherung wird beabsichtigt, die Merkmale einer Filialstrategie für kleine und mittlere Industrieunternehmen zu identifizieren.

\section{Introduction}

Pour nombre de PMI, l'internationalisation est perçue comme une manœuvre stratégique d'extension de l'activité dès lors que la spécialisation sur le marché national ne suffit plus à assurer leur développement ou à garantir leur survie. Dans ce contexte, l'internationalisation se réalise suivant la manœuvre stratégique de spécialisation, les PMI cherchant alors à étendre leurs activités sur des marchés nouveaux avec leurs produits actuels.

De nombreuses études (Cavusgil, 1984 ; Julien et al., 1996) ont analysé cette problématique des manœuvres stratégiques des PMI dans un contexte d'environnements fortement concurrentiels. En revanche, les manœuvres stratégiques internationales des PMI évoluant en environnement à dominante institutionnelle restent à explorer. Considérant l'hypothèse de la prédominance des facteurs institutionnels 
locaux sur les aspects concurrentiels dans des environnements tels que les pays en transition, nous chercherons à mettre en évidence les transformations des stratégies des PMI internationalisées dans ces pays.

La complexité du processus d'internationalisation et le caractère volontairement inductif de nos travaux nous ont conduit à adopter une démarche exploratoire de 30 mois auprès d'une PMI du secteur des matériels de travaux publics routiers. Cette approche longitudinale a abouti à la construction d'une monographie permettant d'apprécier les évolutions de la stratégie internationale de l'entreprise et d'en mesurer les implications. Il en ressort une transformation de la stratégie, conduisant à une extension de ses manœuvres, depuis la spécialisation initiale vers une manœuvre stratégique de filière, dont nous identifierons les principaux critères. La conduite de telles transformations stratégiques étant liée au contexte dans lequel les PMI s'internationalisent, nous soulignerons enfin les facteurs environnementaux propices au développement de telles stratégies.

\section{Le développement international des PMI}

\subsection{Conditions du développement international}

Une revue des travaux sur le management international de l'entreprise fait apparaître une distinction entre l'entreprise en voie d'ouverture à l'étranger ou engagée dans une démarche d'internationalisation de ses activités et l'entreprise mondialisée. La mondialisation est la conséquence du processus d'évolution de grandes firmes passées d'entreprises nationales à des multinationales intégrées, puis diversifiées pour devenir enfin des entreprises mondiales (Chandler, 1986). La nécessité d'un retour sur investissement rapide des capitaux investis dans l'outil industriel et le coût croissant de la R-D, causes et conséquences de la diminution du cycle de vie des produits, obligent les entreprises mondiales à opérer sur des marchés transnationaux, seuls à même d'absorber des volumes de production élevés.

Face à ces entreprises de plus en plus mondialisées, dont les capacités (productives, financières, humaines, etc.) ne cessent de s'accroître, les PMI soucieuses de développer une activité internationale doivent trouver une solution leur permettant d'exister dans l'ombre des grandes entreprises. Fernandez et Noël (1994) envisagent trois possibilités pour ces PMI qui doivent composer avec la présence d'une entreprise mondialisée dans leur environnement : être fournisseur, distributeur ou concurrent direct ; ce dernier choix nécessite toutefois la mise en œuvre de stratégies permettant d'éviter un affrontement direct avec les grandes entreprises. 


\subsection{Les stratégies de développement international}

La différenciation des produits par rapport à la concurrence et plus encore la spécialisation sont présentées comme les deux principales manœuvres stratégiques génériques ${ }^{1}$ pratiquées par les PMI françaises (Ministère de l'Industrie, 1993). Ces stratégies de « renforcement de l'innovation » (Saporta, 1997) apparaissent comme les plus aptes à créer un potentiel de survie dans un environnement concurrentiel fort.

Cette spécialisation de l'activité peut se réaliser suivant deux modalités (Bréchet, 1996) : d'une part, le développement par expansion sur la base du métier actuel, qui emprunte des voies fondées sur des diversifications commerciales par une politique de clientèle ou de gamme; d'autre part, la focalisation ou stratégie de niche, qui vise à privilégier des couples métiers-missions viables et adaptés aux attentes des clients. «Du point de vue de l'offre, il s'agit d'effectuer un choix de métier susceptible de doter l'entreprise d'une capacité concurrentielle suffisante afin de se développer ou de se maintenir. Les stratégies de niche peuvent correspondre soit à un retranchement sur un couple métier/mission existant, soit à une resegmentation du secteur, créatrice d'un nouveau couple métier/mission » (Bréchet, 1996, p. 143) dans le cadre d'une stratégie de développement.

Si l'exploitation de ces stratégies sur le marché domestique ne permet pas d'assurer la survie ou le développement de l'entreprise, l'internationalisation et la diversification peuvent être vues comme des choix stratégiques de développement. Plus particulièrement, « le développement international est envisagé pour beaucoup de PME comme le développement naturel de leur choix d'une stratégie de spécialisation étroite » (Saporta, 1997, p. 3115).

Certains auteurs (Ansoff, 1989; Thérin, 1995) rapprochent l'internationalisation pour les PMI à une forme de diversification; cette diversification géographique est alors considérée comme une manœuvre stratégique générique au même titre que la diversification métier.

À l'inverse, Calori et Harvatopoulos (1988) et Détrie et al. (1997) situent la diversification dans le développement de l'entreprise, après la pénétration du marché traditionnel suivant, d'une part, une stratégie de spécialisation et, d'autre part, l'expansion géographique. Dans cette perspective, les ressources limitées des PMI expliquent l'obligation de choisir entre spécialisation internationale et diversification produit (Saporta, 1997), le développement simultané des deux stratégies s'avérant difficile.

1. «Les manœuvres stratégiques génériques sont constituées de toutes les grandes décisions de base de l'entreprise touchant son activité [donc ses extrants] par rapport à son environnement actuel ou futur, lui assurant ou devant lui assurer sa pérennité par réalisation d'investissements substantiels [positifs ou négatifs] exigeant ou libérant des moyens de production. » (Paturel, 1997)

Revue internationale P.M.E., vol. 14, n 1, 2001 
La spécialisation apparaît donc comme la manœuvre stratégique privilégiée des PMI en contexte international. Dès qu'une PMI dispose d'un savoir-faire spécifique lui permettant d'acquérir une niche sur le plan national, le développement de l'activité privilégie dans un premier temps l'extension de la surface potentielle de clients pour ce savoir-faire, de préférence à l'offre de nouveaux savoir-faire auprès des clients actuels. Liouville et Nanopoulos (1998, p. 44) résument ainsi cette position: «les entreprises spécialisées ont a priori le monde pour marché ou au moins un marché international ». Cette stratégie de spécialisation n'est pas incompatible avec d'autres manœuvres stratégiques, en particulier la domination par les coûts et la différenciation des produits (Paturel et Levet, 1997). Les PMI peuvent ainsi opter pour une stratégie de différenciation visant à se positionner sur des créneaux négligés par les entreprises géantes (Liouville et Nanopoulos, 1998). Dans ce contexte de niche, les PMI misent sur la différenciation de leurs produits pour leur procurer un avantage concurrentiel, et ce, quel que soit leur degré d'implication dans des stratégies d'internationalisation. Seule la nature de cette différenciation varie selon la portée de l'engagement, tandis que les firmes réactives s'appuient sur une supériorité technique du produit et pratiquent des prix élevés, dans une logique de rentabilité immédiate, tandis que les firmes ayant une approche plus proactive de leur internationalisation misent sur une combinaison de facteurs centrés sur la qualité, la performance, l'innovation et un prix concurrentiel (Julien et al., 1996).

Cette logique de différenciation s'inscrit dans les perspectives récentes issues de travaux à propos de la mondialisation. Historiquement, le «village global » devait conduire à une préférence universelle pour des produits normalisés à des prix raisonnables (Levitt, 1983), selon un processus qualifié par Ohmae (1991) de «californisation des besoins ». Seuls quelques produits correspondent effectivement à cette vision. Dans la majorité des situations, l'ouverture des marchés n'exclut pas le maintien, voire l'accroissement des différences entre espaces économiques imparfaitement décloisonnés, d'où la nécessité de suivre la maxime : «Think global, act local ».

Le choix majoritaire des PMI pour aborder les marchés internationaux se faisant suivant une logique de spécialisation sur les savoir-faire existants (Thérin, 1995), de fait, la diversification apparaît comme une manœuvre peu utilisée par ces mêmes PMI. La diversification de savoir-faire semble écartée pour des questions liées aux ressources mobilisables : «les PME qui exportent le plus sont celles qui sont le moins diversifiées en termes de savoir-faire. On peut supposer qu'elles se sont focalisées sur certains marchés et n'ont pas étendu leur domaine de compétences » (Thérin, p. 135). De la même manière, la diversification sectorielle semble peu pratiquée en raison de la difficulté à maîtriser l'information et donc, la connaissance des occasions d'affaires sur différents marchés (Czinkota et Johnston, 1983).

Dans un contexte de mondialisation des échanges, la diversification des activités semble ne concerner que les grandes entreprises, seules entités disposant des ressources suffisantes pour ne pas être obligées de s'en tenir à une seule activité.

Revue internationale P.M.E., vol. 14, nº 1, 2001 
Cette diversification permet alors de « rechercher des synergies entre les activités qui doivent se concrétiser en termes de coûts par des économies de champ » (Liouville et Nanopoulos, 1998, p. 34), indispensables dans un contexte de concurrence par les coûts.

Cette analyse se vérifie dans nombre d'études traitant des manœuvres stratégiques génériques des PMI en environnement international. Mais qu'elles traitent du cas des sous-traitants de l'aéronautique (Thérin, 1995), d'un vaste ensemble de PME françaises et allemandes (Paturel et Levet, 1997), des PMI allemandes et italiennes (Liouville et Nanopoulos, 1998) ou encore des PMI de diverses filières industrielles canadiennes (Julien et al., 1996), toutes analysent les stratégies d'internationalisation au regard de l'environnement concurrentiel international dans son acception la plus courante. Les PMI allemandes, italiennes et françaises évoluent au sein de l'Union européenne, tandis que les PMI canadiennes sont vues dans une perspective nord-américaine. Ces environnements concurrentiels sont complexes, accessibles, turbulents et soumis à une concurrence vive, qu'elle émane des multinationales ou d'entreprises locales. L'agressivité concurrentielle conduit les PMI à se spécialiser davantage afin de se protéger «par la recherche d'une niche, d'un créneau salvateur » (Marchesnay, 1992). Tous les environnements internationaux ne reprennent pas ces caractéristiques concurrentielles. Ainsi, Donckels et Lambrecht (1995) soulignent le rôle spécifique des conditions des environnements propres aux pays en voie de développement dans l'internationalisation de PME européennes. Khanna et Palepu (1997) soulignent, pour leur part, la prédominance des conditions de l'environnement local sur l'aspect concurrentiel dans la formation des stratégies des entreprises évoluant dans les pays émergents.

\section{Le cadre de la recherche}

\subsection{Problématique}

La revue de la littérature sur l'internationalisation des PMI faisant apparaître la prééminence de la stratégie de spécialisation dans les environnements concurrentiels ouverts, notre problématique cherche à vérifier si, dans un contexte environnemental différent, en l'occurrence celui des pays en transition marqué par une domination $\mathrm{du}$ «contexte institutionnel local» sur la notion de «concurrence», les PMI s'appuient toujours sur leur stratégie de spécialisation ou recourent à d'autres manœuvres stratégiques génériques pour assurer leur développement international dans ces pays.

Dans le cadre d'une approche exploratoire, telle que nous l'avons adoptée, la problématique de la recherche se construit et évolue à travers les interactions entre chercheur et acteurs du terrain étudié (Le Moigne, 1995; Thiétart et al., 1999). Initialement, notre objet de recherche était l'offre produit de la PMI et son évolution

Revue internationale P.M.E., vol. 14, n 1, 2001 
au regard des spécificités des marchés des pays en transition. Au fur et à mesure de la construction du corps de connaissances et des observations réalisées, la problématique s'est élargie de l'étude de l'offre produit à celle des manœuvres stratégiques génériques en contexte international. Tenant compte de ces évolutions liées au contexte longitudinal et exploratoire de la recherche, nos hypothèses de travail se traduisent par les propositions suivantes :

Proposition initiale : l'appréhension des marchés industriels des pays en transition par les PMI, dans le cadre de leur développement international, passe par un élargissement de l'offre produit aux conditions spécifiques des pays en transition.

Proposition évoluée : l'évolution de l'offre produit pour s'adapter aux spécificités du contexte conduit les PMI à dépasser la spécialisation, stratégie internationale caractéristique, pour s'orienter vers une stratégie de filière visant à en maîtriser les secteurs clés.

\subsection{Démarche méthodologique}

Le développement international d'une entreprise est un processus long et complexe qui peut être marqué par de nombreuses évolutions, sinon de transformations radicales; outre les facteurs propres à l'entreprise, l'incertitude environnementale est souvent à l'origine de ces changements. Dans des contextes environnementaux par essence aussi instables et évolutifs que les pays en transition eux-mêmes, ce risque ne peut que s'accentuer.

L'approche synchronique, qui caractérise la majorité des études en stratégie (Ageron, 1998), y compris en stratégie internationale, semble inadaptée à l'étude de ce processus. En effet, comme le soulignent Welch et Luostarinen (1988), «le fait de n'étudier que l'activité actuelle sur des marchés étrangers occulte un ensemble de changements internes à la PMI ». À l'inverse, les études longitudinales ou diachroniques permettent de rendre compte du processus d'exportation comme phénomène construit, d'exprimer le contexte dans lequel se produisent les événements et de percevoir les évolutions du processus. Elle garantit enfin un rendu fiable des données par une collecte réalisée «sur le vif » et confortée par des entretiens tout au long du processus. L'étude réalisée a posteriori ne permettrait pas de garantir une telle fiabilité des données recueillies en raison du caractère sélectif de la mémoire des acteurs dès que l'on aborde des questions relevant de la stratégie (Plane, 1999).

Une telle approche longitudinale implique une présence physique du chercheur dans une démarche qualitative de type «recherche-action et intervention ». Elle repose sur une forte interaction entre le chercheur et le terrain dans le cadre d'un processus progressif de construction de la connaissance (Martinet et al., 1990). Le manque de données existantes sur la problématique proposée conduit donc à adopter cette approche inductive.

Revue internationale P.M.E., vol. 14, nº 1, 2001 
Le choix de l'entreprise s'est effectué en fonction de critères génériques (avoir un effectif compris entre 20 et 99 salariés $^{2}$, être indépendante au plan capitalistique et réaliser des produits industriels finis) et spécifiques à notre problématique (existence d'un développement international, présence dans les pays en transition). Cela nous a conduit à retenir une PMI indépendante de 65 personnes, fabriquant des matériels de travaux publics destinés à l'entretien des routes.

L'étude a été conduite sur une période de 30 mois, sous la forme :

- d'entretiens semi-directifs approfondis avec les trois principaux responsables de l'entreprise (PDG, directeur commercial et directeur technique). Cinq séries d'entretiens de deux heures ont été réalisées autour de la question du développement international et de la stratégie adoptée (soit 15 entretiens au total). La structure de l'entretien comportait deux parties. L'internationalisation était abordée, d'une part, à travers les objectifs et les réalisations et, d'autre part, à travers les manœuvres stratégiques, les modalités d'internationalisation (et leurs évolutions) et les produits proposés ;

- d'entretiens semi-directifs approfondis avec quatre acteurs russes (deux clients, le responsable de l'entreprise russe participant à la coopération et un acteur institutionnel) en contact avec la PMI lors de deux voyages d'études (soit sept entretiens). La première série d'entretiens, réalisée au cours de l'année de lancement de la démarche internationale de la PMI, portait sur les conditions du marché de la Russie et les attentes des acteurs locaux à l'égard de la démarche engagée par la PMI. Le seconde série, réalisée après deux années de développement, a permis de dresser un premier bilan de l'action engagée et des orientations à prendre ;

- de participation, à trois reprises, aux réunions stratégiques annuelles de la direction de l'entreprise avec son encadrement, en qualité d'observateur.

À partir des données recueillies, nous avons réalisé une monographie de l'entreprise, axée sur sa stratégie internationale avant de confronter ces observations à notre problématique de recherche.

2. Les PMI françaises employant entre 20 et 99 salariés représentent $80,2 \%$ de la population PMI en 1998 (soit 16301 entreprises). En revanche, elles ne représentent que $26 \%$ du volume des exportations réalisées par l'ensemble des PMI. Les entreprises de 100 à 499 salariés, au nombre de 4015 (19,8\%) regroupent $74 \%$ des exportations. Dans un cadre plus général, l'étude réalisée s'inscrivant dans une optique de développement des stratégies internationales des PMI françaises, nous nous sommes plus particulièrement intéressé aux entreprises pour lesquelles ce développement reste à accomplir. (Source : SESSI, 1998) 


\section{Résultats de la recherche}

\subsection{Les conditions du développement international de la PMI}

L'entreprise objet de l'étude est spécialisée dans un marché, le secteur des travaux publics routiers et dans un produit, les matériels pour la réalisation d'enduits superficiels. Historiquement fondé sur une innovation technologique de rupture, son avantage concurrentiel s'est déplacé progressivement vers une forte capacité de différenciation de ses produits par une adaptation aux demandes du client autour du concept central qui demeure. La connaissance technique des clients, les conditions particulières de chaque route entretenue (relief, climat, etc.) et la complexité technique du produit sont à l'origine de cette stratégie de différenciation. La concurrence limitée et les gains liés à la différenciation rendant cette activité profitable sur le marché national, la PMI s'est spécialisée dans ce segment du marché.

Antérieurement au choix du développement international dans les pays en transition, la stratégie internationale de la PMI se caractérisait par une vision opportuniste des marchés étrangers (Julien et al., 1996), tirant profit d'occasions d'affaires, dans un souci de rentabilité immédiate.

Pour assurer son développement, la PMI a choisi de s'en tenir à son activité spécialisée et d'en assurer l'extension géographique. Le choix des pays en transition résulte d'une analyse de la situation locale qui révèle l'importance du réseau de communication routier dans la réussite de la transition d'une économie planifiée vers une économie de marché (Mercier-Suissa, 1998). Le décalage entre le discours des autorités locales qui soutiennent le développement de cette activité (Larçon, 1996) et la réalité de la situation fait ressortir d'importantes disparités, qui sont autant d'occasions d'affaires. À la politique de grands travaux de l'époque soviétique succède en effet, depuis 1991, un abandon de la maintenance routière. Outre la dégradation du réseau, qui freine le développement des échanges, cette situation est marquée par la disparition des firmes fabriquant des matériels de travaux publics routiers et une perte de savoir-faire des opérateurs dans la maintenance routière. La réfection du réseau de communication et la reconversion des industries locales sont des enjeux importants pour les acteurs institutionnels locaux. Ces derniers pourront ainsi être amenés à agir afin de favoriser l'implication de firmes étrangères dans ces processus de transformation. Pour saisir ces occasions d'affaires, il est nécessaire de répondre à la triple problématique du coût des investissements en période de récession budgétaire, du choix de techniques routières adaptées et reconnues par les décideurs locaux et de la demandeinstitutionnelle - d'implication des acteurs industriels locaux dans la démarche.

Conclusion 1. Les pays en transition ont un besoin important d'entretien des routes. Il n'existe pas sur ces marchés de matériels adaptés aux conditions économiques et techniques locales permettant d'engager une politique de maintenance routière adéquate. Les produits fabriqués par la PMI peuvent répondre à ces besoins. 


\subsection{L'évolution de l'offre produit}

Aux premières offres commerciales de la PMI sur les marchés des pays en transition, les utilisateurs potentiels ont fait valoir les conditions locales spécifiques, tant économiques que techniques. Pour répondre à ces demandes, la PMI a développé un produit adapté. Ainsi, la géographie marquée par de grandes étendues planes, par opposition au tortueux réseau français, impose des matériels de grande capacité. De même, les conditions climatiques rigoureuses conduisent à revoir le matériel tel qu'il est conçu pour d'autres marchés, en termes d'équilibre performances-fiabilité.

En revanche, la contrainte économique, qu'elle concerne le coût d'investissement du matériel ou le coût de mise en œuvre est, dès l'origine, intégrée dans l'offre produit. Les matériels fabriqués par la PMI représentent en effet un coût d'investissement modéré et la technique qu'ils servent est reconnue comme l'une des plus économiques pour l'entretien des routes.

Conclusion 2. Les produits fabriqués par la PMI pour satisfaire aux conditions du marché des pays en transition nécessitent « une standardisation adaptée » (Machkova, 1998, p. 110). Celle-ci doit obéir à une double contrainte : 1) répondre en termes de produit aux exigences locales, tant économiques que techniques; 2) satisfaire aux contraintes d'applications routières, c'est-à-dire aux résultats qualitatifs et financiers de l'exploitation de ces matériels.

Les premières ventes de matériels, après un an de négociations, font apparaître une réalité aux dirigeants de la PMI : les techniques de travaux correspondant à l'usage des matériels fabriqués sont connus des décideurs, mais leur mise en œuvre en conditions réelles est méconnue au niveau des chantiers. Ce constat confirme l'existence dans les pays en transition d'un décalage entre le niveau d'instruction des acteurs des pays en transition (forte culture technique) et la réalité du terrain, qui associe à la connaissance théorique des savoir-faire au service d'un résultat (en l'occurrence le niveau de qualité des travaux), comme l'a relevé Mercier-Suissa (1998).

Les travaux d'entretien des routes nécessitent, en effet, outre une connaissance des matériels, une maîtrise de la technique routière utilisée (en l'occurrence les enduits superficiels) et de l'organisation des chantiers. À défaut de quoi, le risque d'échec dans la réalisation des routes est élevé et pourrait conduire les décideurs locaux à pointer du doigt les matériels de la PMI.

Après avoir intégré, dans un premier temps, une offre d'assistance et de formation à la réalisation de chantiers d'entretien routier, la PMI s'est ensuite engagée directement dans la réalisation de ces chantiers en coopération avec une entreprise russe de travaux publics, d'abord à titre ponctuel, puis sous la forme d'une entreprise commune avec une implication directe dans ses activités. Cette 
intégration d'une activité de travaux publics routiers visait à apporter des références de chantiers aux matériels afin d'en démontrer la qualité et de formaliser une organisation des travaux adaptée aux contraintes locales des pays en transition.

Conclusion 3. Les conditions particulières résultant de la transition (économique, technologique, organisationnelle) rendent nécessaire l'évolution de l'offre de la PMI pour intégrer la formation des opérateurs à la réalisation des travaux utilisant les matériels fabriqués. Elles conduisent surtout à élargir le cadre des activités de la PME, depuis le métier très spécialisé de constructeur de matériels de travaux publics vers des activités situées en aval sur la filière des routes.

L'engagement dans la filière des travaux publics routiers de la PMI par la réalisation de travaux d'entretien des routes amène ses dirigeants à mettre en évidence l'une des faiblesses majeures de cette filière. Outre des matériels et des techniques adaptés ainsi qu'une mise en œuvre des travaux organisée, la réussite d'une politique d'entretien des routes passe par l'existence d'un référentiel de contrôle adéquat, tel qu'il existe dans les principaux pays occidentaux.

Au cours de leur période soviétique, ces pays ont évolué dans un cadre normatif marqué par une formalisation systématique des procédures, des objectifs définis en volumes et un arbitrage centralisé des décisions (Bournois et Louart, 1998). La transition vers une économie de marché déplace la régulation de l'activité vers les entreprises et intègre la notion de résultats économiques des travaux, tant en performance qu'en qualité, éléments relégués au second plan dans l'ancien système (Luthans, Patrick et Luthans, 1995) ${ }^{3}$.

La détérioration de l'ancien système normatif soviétique donne lieu à la création d'un environnement parfois souple, parfois extravagant dans lequel coexistent d'anciennes normes, l'embryon d'une nouvelle réglementation et de nombreux vides. De cette situation, « les entrepreneurs russes disent vivre une période assez étonnante où ils créent leur champ d'action autant qu'ils le subissent » (Bournois et Louart, 1998, p. 76). La transition et l'incertitude qui en résultent permettent à des entreprises de devenir créatrices de normes et de les exporter vers leur environnement (Demil, 1998). Sur la base de ce constat et profitant des occasions qui lui étaient offertes, la PMI s'est impliquée auprès des institutions russes dans les questions réglementaires et de mesure de la performance économique des travaux routiers. Elle a ainsi intégré le comité de normalisation GOST des travaux publics routiers par l'entremise d'un représentant de son entreprise commune.

3. "Before Perestroika, Russia could be characterized as a centrally planned economy with set production targets and products mixes and fixed prices. Performance was measured in terms of meeting government-set production goals - often in tonnage produced ». (Luthans, Patrick et Luthans, 1995, p. 12; c'est nous qui soulignons). 
Par cette activité, la PMI a cherché, d'une part, à créer un référentiel d'évaluation des travaux réalisés afin de réduire les risques d'assimilation d'un échec à la qualité du matériel vendu et, d'autre part, à placer les performances à un niveau suffisamment élevé pour qu'on préfère ses matériels aux productions locales.

Conclusion 4. L'extension de l'offre à des activités de travaux publics conduit à s'intéresser aux conditions de réalisation et de mesure de la performance économique de ces travaux et donc, à accroître l'engagement de la PMI dans une activité située dans la filière des travaux publics routiers très en aval de sa position initiale.

Parallèlement à ce processus visant à assurer la maîtrise des travaux réalisés avec ses matériels, la PMI s'est engagée dans une démarche de fabrication locale de ses matériels en partenariat avec un constructeur russe d'outillage de travaux publics. Cette démarche répond à une double demande, celle des clients locaux et celle de l'État: les droits de douanes élevés sur les produits finis poussent les clients locaux à choisir des matériels d'origine nationale, même si leur niveau de performance est inférieur.

L'organisation industrielle issue de l'économie planifiée fait apparaitre des structures complexes (Paturel, 1995). Ainsi, il existe des liens forts entre les entreprises de travaux publics et les unités de production de matériels de travaux publics ; la situation économique de ces dernières étant de loin la moins enviable. L'État, souvent directement associé à ces entités, veut tout autant entretenir ses routes que relancer ses unités de fabrication. Outre des problèmes d'organisation interne, le principal problème de ces unités réside dans les difficultés d'approvisionnements en composants industriels ; difficultés qu'expliquent la disparition du Gosnab ${ }^{4}$ et l'émergence dans les activités de négoce d'un grand nombre d'intermédiaires qui n'apportent aucune valeur ajoutée aux produits et en grèvent considérablement les prix.

Pour répondre à cette double demande, la PMI a établi un partenariat de transfert de technologie avec une unité industrielle russe. Plus que la cession de licence réalisée, l'intérêt de cet accord pour notre problématique réside dans le rôle de grossiste joué par la PMI. Celle-ci vend à son partenaire des composants industriels achetés en France (y compris pour ses propres fabrications) à un prix de marché. Les composants industriels, faiblement taxés à l'importation, sont de fait achetés par l'entreprise russe à la moitié du prix de ceux qu'elle pourrait se procurer directement en Russie.

Conclusion 5. Indépendamment des manœuvres stratégiques pratiquées et en dehors de toute transformation du produit, le développement international de la PMI en Russie passe par des modalités coopératives (transfert de technologie) afin

4. Organisme d'État chargé de la gestion des approvisionnements des entreprises sous le régime soviétique.

Revue internationale P.M.E., vol. 14, n 1, 2001 
de respecter les contraintes et de répondre aux demandes des acteurs locaux. Cette modalité d'internationalisation conduit la PMI à développer le métier de grossiste en composants industriels, activité située en amont de son métier de base.

\subsection{L'impact de la transformation de l'offre sur l'activité de la PMI}

On ne saurait conclure cette monographie sans évoquer les résultats finals, au terme de 30 mois de développement international dans les pays en transition et, plus particulièrement en Russie. La PMI, en dépit de la crise de l'été 1998, a réussi son développement international comme en attestent ses résultats (tableau 1). Mais, plus encore que le volume de matériels vendus, c'est en termes d'intégration dans le contexte russe que se situe la plus grande réussite de la PMI. En effet, ses dirigeants, comme les acteurs locaux, considèrent qu'elle fait partie intégrante de la filière locale des travaux publics routiers. Sa participation au niveau fédéral à l'établissement des cahiers des charges d'appels d'offre sur les matériels de travaux publics routiers (y compris certains ne relevant pas de son secteur d'activité) démontre son degré d'intégration.

\section{TABLEAU 1}

Présentation de l'activité de la PMI en Russie

\begin{tabular}{|c|c|c|c|}
\hline & 1997 & 1998 & 1999 \\
\hline Nature de l'offre & - Vente de matériels & $\begin{array}{l}\text { - Vente de matériels } \\
\text { - Réalisation de chantiers } \\
\text { d'entretien des routes }\end{array}$ & $\begin{array}{l}\text { - Vente de matériels } \\
\text { - Réalisation de } \\
\text { chantiers d'entretien } \\
\text { des routes } \\
\text { - Normalisation }\end{array}$ \\
\hline Secteurs d'activité & $\begin{array}{l}\text { - Construction } \\
\text { mécanique }\end{array}$ & $\begin{array}{l}\text { - Construction mécanique } \\
\text { - Travaux publics }\end{array}$ & $\begin{array}{l}\text { - Construction } \\
\text { mécanique } \\
\text { - Travaux publics }\end{array}$ \\
\hline $\begin{array}{l}\text { Modalités } \\
\text { d'internationalisation }\end{array}$ & - Exportation & $\begin{array}{l}\text { - Exportation } \\
\text { - Coopération puis } \\
\text { entreprise commune }\end{array}$ & $\begin{array}{l}\text { - Exportation } \\
\text { - Coopération puis } \\
\text { entreprise commune }\end{array}$ \\
\hline Manœuvre stratégique & Spécialisation & Stratégie de filière & Stratégie de filière \\
\hline Chiffre d'affaires réalisé & $7 \mathrm{MF}$ & $12 \mathrm{MF}$ (avant la crise) & $17 \mathrm{MF}$ \\
\hline $\begin{array}{l}\text { Part du chiffre d'affaires } \\
\text { total }\end{array}$ & $15 \%$ & $24 \%$ & $26,5 \%$ \\
\hline $\begin{array}{l}\text { Salariés impliqués } \\
\text { dont Russie }\end{array}$ & $\begin{array}{l}8 \\
0\end{array}$ & $\begin{array}{r}17 \\
7\end{array}$ & $\begin{array}{l}29 \\
20\end{array}$ \\
\hline
\end{tabular}




\section{Vers l'émergence d'une stratégie de filière}

\subsection{De la spécialisation à l'extension des activités}

Les transformations successives de l'offre de la PMI pour s'adapter aux spécificités de l'environnement conduisent à appréhender de manière différente sa stratégie d'internationalisation. Au fur et à mesure que la PMI découvre le marché des pays en transition et les déterminants locaux qui l'animent, sa stratégie perd ses caractéristiques issues d'un développement en environnement à dominante concurrentielle (tableau 2).

\section{TABLEAU 2}

\section{Évolution de la stratégie de la PMI en relation avec l'environnement}

\begin{tabular}{|c|c|c|}
\hline $\begin{array}{l}\text { Situation } \\
\text { de l'environnement }\end{array}$ & $\begin{array}{l}\text { Au niveau des activités } \\
\text { de la PMI }\end{array}$ & $\begin{array}{l}\text { Au niveau de la stratégie } \\
\text { de la PMI }\end{array}$ \\
\hline $\begin{array}{l}\text { Besoins à court terme } \\
\text { et importants de travaux } \\
\text { de maintenance routière }\end{array}$ & $\begin{array}{l}\text { Choisir l'internationalisation } \\
\text { sur ces marchés: } \\
\text { extension des débouchés } \\
\text { des produits fabriqués }\end{array}$ & $\begin{array}{l}\text { Développement de la } \\
\text { spécialisation de la PMI: } \\
\text { nouveaux marchés pour les } \\
\text { produits actuels }\end{array}$ \\
\hline $\begin{array}{l}\text { Existence de conditions locales } \\
\text { spécifiques pour la réalisation } \\
\text { des travaux routiers }\end{array}$ & $\begin{array}{l}\text { Adapter des matériels } \\
\text { aux conditions locales: } \\
\text { «standardisation adaptée » }\end{array}$ & $\begin{array}{l}\text { Différenciation } \\
\text { au service de } \\
\text { la spécialisation }\end{array}$ \\
\hline $\begin{array}{l}\text { Mauvaise organisation des } \\
\text { chantiers de maintenance } \\
\text { routière: risque élevé d'échec } \\
\text { des travaux }\end{array}$ & $\begin{array}{l}\text { Assurer la maîtrise des travaux } \\
\text { réalisés avec les matériels } \\
\text { vendus : réalisation } \\
\text { des chantiers }\end{array}$ & $\begin{array}{l}\text { Diversification } \\
\text { aval* }\end{array}$ \\
\hline $\begin{array}{l}\text { Manque d'appréciation } \\
\text { de la notion de performance } \\
\text { économique des travaux routiers: } \\
\text { risque élevé d'échec de la } \\
\text { politique de maintenance }\end{array}$ & $\begin{array}{l}\text { Proposer des critères } \\
\text { d'appréciation de la performance } \\
\text { économique des travaux routiers: } \\
\text { participation au comité de } \\
\text { normalisation }\end{array}$ & $\begin{array}{l}\text { Extension de la } \\
\text { diversification aval }\end{array}$ \\
\hline $\begin{array}{l}\text { Manque de compétitivité } \\
\text { des entreprises locales de } \\
\text { matériels de travaux publics } \\
\text { dû au besoin de réorganisation } \\
\text { des activités et au coût d'achat } \\
\text { prohibitif de composants } \\
\text { industriels performants }\end{array}$ & $\begin{array}{l}\text { Développer le marché par } \\
\text { une production locale: } \\
\text { partenariat de fabrication fondé } \\
\text { sur le transfert de technologie } \\
\text { et la vente de composants } \\
\text { industriels }\end{array}$ & Diversification amont \\
\hline
\end{tabular}

* «La diversification est une manœuvre ayant pour effet d'accroître la diversité des activités poursuivies par l'entreprise. La diversification stratégique nécessite l'acquisition ou l'apprentissage de compétences nouvelles. Lorsque le développement de produits ou de marchés nouveaux se fait sans qu'il y ait changement de métier, il s'agira de diversification commerciale.» (Koenig, 1996)

Lorsque l'environnement concurrentiel n'est pas le principal facteur susceptible d'influencer la stratégie des entreprises, comme c'est encore le cas dans les pays en transition, la spécialisation en tant que manœuvre stratégique générique 
privilégiée des PMI perd son intérêt. En effet, l'objet de la stratégie n'est plus de se créer un «créneau salvateur » permettant d'exister dans un cadre concurrentiel où s'affrontent multinationales, PME mondialisées et PME locales (Fernandez et Noël, 1994). Plutôt que de renforcer son pré carré, l'entreprise doit s'ouvrir à l'environnement pour en percevoir les besoins. Dans ces pays, la concurrence, qu'elle soit locale ou internationale, reste limitée. D'un côté, les entreprises locales vivent difficilement la transition économique et, de l'autre, les multinationales se sont positionnées essentiellement sur les marchés de grande consommation et quelques marchés industriels, généralement liés aux matières premières. La majorité des secteurs industriels présentent un intérêt limité au regard des faibles moyens de l'État et des niveaux de risques difficilement acceptables.

L'étude longitudinale présentée précédemment confirme cette analyse. En effet, la PMI s'est engagée dans un premier temps en Russie dans le cadre d'une stratégie de spécialisation ayant pour objet de satisfaire l'unique marché des matériels pour l'entretien des routes. En dépit d'un potentiel important, ce marché peut être qualifié de niche du fait de son faible volume actuel, d'une concurrence réduite et de l'existence d'un nombre limité de technologies applicables. Initialement, l'adaptation de l'offre produit aux contraintes locales (conclusion 2) marque la volonté de la PMI de s'orienter vers une stratégie de différenciation afin de préserver sa niche de marché de la concurrence et ainsi consolider son activité spécialisée.

Les conditions particulières de l'environnement des pays en transition amènent cependant à revoir les facteurs clés de succès de la stratégie internationale de la PMI (conclusion 3). En effet, les facteurs traditionnellement retenus pour assurer sa compétitivité dans son environnement national soumis à de fortes turbulences, à savoir le caractère innovant des produits proposés et les performances qui y sont associées, leur adaptation aux conditions d'utilisation locales, qui assure une différenciation par rapport à l'offre concurrente, et, enfin, la maîtrise des coûts permettant de proposer un prix compétitif, ne garantissent pas le succès de l'internationalisation dans les pays en transition. Le constat de l'échec des premiers travaux, pourtant réalisés avec des matériels éprouvés et adaptés aux conditions locales, révèle la nécessité de tenir compte des contraintes locales, en particulier de celles de la filière des travaux publics, beaucoup plus que de celles issues de l'environnement concurrentiel.

La problématique stratégique des marchés de pays en transition ne relève pas uniquement du positionnement par rapport à la concurrence, car les besoins de l'environnement local ne s'expriment pas seulement en termes de compétitivité des produits achetés. La stratégie de la PMI se doit d'intégrer ces besoins pour garantir la pérennité de son internationalisation. Autant que la demande de produits performants, la transition du système planifié vers une structure économique de marché a mis en lumière la nécessité de recourir à des organisations et des processus adaptés 
aux nouvelles contraintes économiques. Devant ces nouvelles contraintes, la PMI doit dépasser sa stratégie de spécialisation pour proposer une offre apportant des solutions à l'ensemble de la filière d'activité dans laquelle elle évolue.

Dans un contexte de filière industrielle, l'offre de la PMI s'inscrit dans un ensemble complexe d'activités complémentaires, articulées au sein d'un système productif, qui s'enchaînent pour aboutir à un résultat, c'est-à-dire un produit fini. L'ensemble des opérations intermédiaires de transformation ou d'apport de valeur ajoutée ou de technologie se place donc dans une cohérence finalisée. Au sein de ce séquençage apparaissent certaines opérations, mobilisant plus de ressources ou plus fortement créatrices de valeur ajoutée. La maîtrise de ces opérations clés est indispensable à la cohérence globale de l'activité et à la qualité du produit final ; et corollaire de cette exigence, les acteurs maitrisant ces opérations jouent un rôle central dans la filière.

Ainsi, dans la filière française des travaux publics routiers, les opérations clés se situent très en amont dans la filière au niveau des matériaux de base que sont le bitume et les gravillons. Des facteurs politiques et réglementaires liés à l'exploitation de ces ressources naturelles expliquent cette donnée et font des pétroliers et carriers les acteurs incontournables de la filière des travaux publics; de fait, leur influence s'étend sur l'ensemble de la filière. C'est ainsi qu'on retrouve des sociétés telles que BP impliquées dans la réalisation du produit final qu'est la route.

Dans ce contexte, les autres opérateurs, fournisseurs d'équipements d'exploitation ou d'application, terrassiers, etc., doivent s'adapter pour s'intégrer au processus sans en dénaturer la qualité. Devant la puissance des opérateurs clés, ils développent des stratégies de spécialisation.

Dans les pays en transition, c'est au niveau des matériels et des technologies de réalisation des chaussées que se situent les opérations clés de la filière des routes. La transition rend nécessaire la mise en place de nouvelles techniques d'entretien économiques des routes, que les opérateurs locaux ne maîtrisent pas. Ces techniques étant liées à des matériels particuliers, il est possible pour un constructeur d'étendre sa maîtrise sur ce marché en intégrant à son offre la mise en œuvre de ses propres matériels. Associée à cette méconnaissance des techniques, le défaut de repères dans la mesure de la performance économique permet en fin de chaîne de maîtriser l'ensemble de la filière.

Autre élément clé de la filière, la volonté de reconvertir l'industrie locale dans la fabrication de matériels de travaux publics permet d'intervenir en amont du processus de production en verrouillant les approvisionnements en composants industriels.

Là où une présence sur l'ensemble de la filière des travaux routiers (depuis l'extraction des matériaux jusqu'à la réalisation des routes) nécessiterait des ressources importantes que seule une entité de grande taille pourrait assumer, la PMI

Revue internationale P.M.E., vol. 14, n 1, 2001 
a su mettre à profit les conditions environnementales des pays en transition pour s'assurer la maîtrise des points clés de la filière. Le recours à un partenaire local, dans le cadre d'une entreprise commune, a permis à la PMI de s'intégrer dans les activités aval de la filière en limitant les investissements aux seuls matériels. La main-d'œuvre, à la fois formée et bon marché, ainsi que les matériaux facilement disponibles relevaient du partenaire local. Ce même partenaire, conscient de l'intérêt d'une coopération favorisant sa propre reconversion industrielle, a apporté dans la balance sa connaissance des réseaux institutionnels locaux, indispensable pour accéder à l'information pertinente, « ce réseau de relations étant l'armature classique et fondamentale de tout développement commercial en Russie » (Mercier-Suissa, 1998, p. 84). En dépit d'un engagement limité de ressources, elle est devenue un acteur essentiel de la filière en apportant des réponses aux contraintes subies par les acteurs locaux.

\subsection{Caractéristiques d'une stratégie de filière dans le cas des PMI}

Sur la base des constats relatifs à la filière des travaux publics routiers, l'approche exploratoire adoptée dans la monographie nous conduit à proposer certaines caractéristiques des marchés internationaux susceptibles de favoriser le développement de stratégies de filière par les $\mathrm{PMI}^{5}$ :

- l'absence d'un acteur dominant l'ensemble de la filière. Dès qu'un acteur ou une catégorie d'acteurs maîtrise l'ensemble des activités de la filière, comme cela se produit dans les Travaux publics en France, les autres acteurs se trouvent en situation de dépendance et n'ont d'autre choix que de se spécialiser et d'assurer leur développement par une extension des marchés à l'international (Marchesnay, 1992);

- l'acceptation par l'environnement de technologies de substitution. La filière ne doit pas être hermétique aux technologies alternatives - comme c'est le cas dans la filière agricole au sein de l'Union européenne à propos des organismes génétiquement modifiés - sur lesquelles reposent les points de rupture permettant à une entreprise de s'immiscer. Concernant les OGM, technologie de substitution aux techniques traditionnelles, autant que la question de la résistance aux pesticides, c'est la maîtrise des semailles (grains stériles) en amont de la filière qui est le facteur stratégique clé. C'est précisément ce point qui est l'enjeu des débats actuels ;

5. Par-delà l'approche exploratoire visant à identifier ou clarifier des phénomènes inconnus ou méconnus, les caractéristiques relevées devront faire l'objet d'une confirmation sur un champ d'étude plus vaste, tant en termes de secteur d'activité que de pays concernés. 
- des turbulences environnementales et institutionnelles qui prennent le pas sur la notion de concurrence. C'est particulièrement vrai dans les pays en transition où les mutations font apparaître de manière générale une défaillance du système d'approvisionnement et/ou du processus de fabrication local; le manque de maîtrise des techniques par les utilisateurs et, enfin, l'inadaptation des structures réglementaires et de leur capacité de régulation.

TABLEAU 3

Le modèle de la stratégie de filière

\begin{tabular}{lll}
\hline & Stratégie de spécialisation & Stratégie de filière \\
\hline Environnement & turbulent concurrentiel & turbulent institutionnel \\
\hline Besoin & compétitif (prix) & organisationnel \\
\hline Positionnement sur une filière & focalisation sur un segment & présence aux points clés \\
\hline Produit & différencié & $\begin{array}{l}\text { multiples et intégrés sur } \\
\text { différents points de la filière }\end{array}$ \\
\hline Relations à l'environnement & de transaction (marché) & de coopération \\
\hline Finalité & maîtrise d'une niche de marché & $\begin{array}{l}\text { maîtrise d'une application } \\
\text { industrielle }\end{array}$ \\
\hline
\end{tabular}

Ces différents facteurs se retrouvent dans de nombreux secteurs d'activité des pays en transition, mais aussi dans d'autres pays pour des technologies qui ne sont pas forcément maîtrisées au niveau de l'industrie nationale. C'est par exemple le cas - aussi paradoxal qu'il puisse paraître - de la gestion du contrôle aérien et de la traçabilité aéroportuaire aux États-Unis. Dans un contexte réglementaire difficile, car lié à des questions de sécurité, il est apparu qu'aucun acteur local n'avait une maîtrise de cette filière ; de plus, il existait des technologies alternatives plus performantes que celles exploitées par les entreprises du secteur. Mettant à profit ce contexte, une PMI française fabricante de terminaux et systèmes de lecture intelligent a su s'insérer dans les points clés de cette filière (Bourcieu, 2000) et dispose d'une position préférentielle sur le marché américain où elle est le seul fournisseur européen d'American Airlines. Sa position actuelle d'intégrateur de la traçabilité aérienne - de l'édition des billets à la traçabilité des bagages en passant par les plans de vols des contrôleurs aériens - en fait un acteur essentiel lors des choix de conception des nouveaux aéroports.

Pour Paturel (1997), les stratégies de filière relèvent certes des manœuvres stratégiques d'intégration verticale amont et aval, mais aussi d'une logique de diversification à travers «de nouvelles perspectives de développement produits /

Revue internationale P.M.E., vol. 14, n 1, 2001 
marchés / technologies en se rapprochant d'autres filières [...] Vu sous cet angle, elle s'intègre à la manœuvre stratégique de diversification selon une logique technologique ${ }^{6}$ avec élargissement de l'espace de compétence de l'entreprise » (p. 106).

Cette approche souligne trois caractéristiques essentielles de la stratégie de filière telle qu'elle pourrait être adoptée par les PMI dans le cadre de leur développement international :

1. Elle se fonde sur une maîtrise technologique apportant un avantage concurrentiel et d'applications sur de nouveaux domaines d'activités stratégiques (DAS). Cette différenciation technologique peut être absolue - maîtrise d'une technologie unique - ou relative - maîtrise d'une technologie inexploitée sur un marché donné, comme dans le cas des pays en transition.

2. Elle conduit à s'intégrer à la filière d'activité des clients plus qu'à sa propre filière. Dans le cas de la monographie, la PMI s'est positionnée sur la filière des travaux publics routiers et non sur celle dont elle est issue, à savoir les constructions mécaniques. La maîtrise d'une technologie qui apparaît complexe vue depuis la filière des clients facilite en effet l'intégration de la PMI. Dès l'instant où elle maîtrise l'un des facteurs clés de la filière de ses clients, la PMI peut essayer d'étendre sa maîtrise sur d'autres facteurs clés. En ce sens, la stratégie de filière adaptée aux PMI se rapproche de la stratégie générique de filière telle qu'elle est présentée par Paturel (1997) lorsqu'il parle de «rapprochement à d'autres filières ». Cette condition implique donc pour les PMI de s'intéresser de près aux activités de leurs clients afin d'être en mesure de saisir les occasions d'affaires.

3. Elle se fonde sur la maîtrise de nœuds technologiques particuliers permettant de trouver de nouveaux DAS (Paturel, 1997). C'est en effet par leur maîtrise d'une technologie spécifique que les PMI peuvent s'insérer dans la filière de leurs clients. Mais, tout autant que la notion de technologie, c'est celle de nœuds ou points clés qu'il s'agit de souligner. En effet, la maîtrise de l'ensemble des activités d'une filière est irréaliste pour une PMI dont les ressources tant humaines que financières sont par définition limitées. De fait, les PMI voulant développer une stratégie de filière doivent en identifier les points clés qu'il s'agira de maîtriser. Ces points sont susceptibles de changer non seulement en fonction de la nature des filières, mais aussi des différents types d'environnements rencontrés (à

6. C'est nous qui soulignons. 
dominante concurrentielle ou institutionnelle). C'est ainsi que dans le cas de la filière des travaux publics routiers dans les pays en transition, nous avons fait ressortir les points clés de la filière au regard:

- de la technologie d'application des matériaux pour les enduits superficiels ;

- de la réalisation des techniques de maintenance routière;

- du référentiel d'évaluation des travaux réalisés ;

- des sources d'approvisionnements en composants industriels.

Il semble donc que la maîtrise des points clés s'exerce sur des nœuds technologiques, mais aussi des nœuds relevant d'autres aspects tels que la normalisation.

Ces trois caractéristiques apparaissent comme les principales conditions de la réalisation de la stratégie de filière par la PMI dans les pays en transition. Elles se caractérisent par l'existence d'une condition préalable, à savoir un nécessaire avantage technologique, générateur d'une avancée importante par rapport à la situation antérieure et la mise en œuvre d'une démarche spécifique, visant à maîtriser les points clés de la filière. Il ne s'agit pas, en effet, pour une PMI de disperser ses ressources «limitées » sur l'ensemble des activités, mais bien de se focaliser sur les points de passage obligés de la filière.

\section{Conclusion}

Khanna et Palepu (1997) ont souligné la nécessité pour les entreprises internationales d'avoir des activités diversifiées (pouvant aller jusqu'au conglomérat) dans les pays émergents, de préférence à la stratégie de focalisation pour laquelle optent généralement les économies de marché. Les facteurs explicatifs de ce choix étaient reliés à la nécessité de répartir les risques liés aux distorsions de l'environnement concurrentiel (inefficacité du système juridique, difficultés d'accès à l'information et interventionnisme faussant le mécanisme de marché), très élevés dans les pays émergents.

Si nos travaux ont cherché à mettre en évidence la transformation de la stratégie internationale de la PMI depuis la focalisation vers la manœuvre stratégique de filière, confirmant ainsi les résultats de Khanna et Palepu, en revanche les déterminants de cette extension de l'activité diffèrent. Bien qu'il faille nuancer la portée des résultats de la recherche, dont le caractère monographique et exploratoire implique une confirmation sur une base d'étude élargie, il apparaît cependant que cette extension de l'activité vise à saisir les occasions d'affaires issues d'environnements où les facteurs institutionnels prennent le pas sur les aspects concurrentiels. La stratégie de filière, telle qu'elle semble se dessiner à travers le cas de cette PMI, ne vise pas à maîtriser tous les stades de la filière, ce qui supposerait à l'évidence des 
besoins disproportionnés de ressources financières, humaines, organisationnelles, etc. Elle se fonderait plutôt sur une implication forte sur les points clés de la filière. L'application de telles manœuvres stratégiques par les PMI en contexte international semble s'inscrire dans une perspective proactive (saisie d'occasions d'affaires) et non comme une réaction de sauvegarde face aux risques issus d'environnements institutionnellement instables. L'émergence de cette relation entre stratégie de filière et proactivité en environnement international, jusqu'ici peu explorée (Cavusgil, 1984 ; Fernandez et Noël, 1994), nous semble une piste de recherche particulièrement féconde.

\section{Bibliographie}

AGERON, B. (1998), Construction d' une capacité de réponse d' une PMI à l' international : approche descriptive et compréhensive à partir du modèle de Bilkey et Tesar, $\mathrm{IV}^{\mathrm{e}}$ Congrès international francophone de la PME, Metz, octobre, 16 p.

Ansoff, I. (1989), Stratégie du développement de l'entreprise, Paris, Les Éditions d'Organisation.

BAKER, M. et S.H. BECKER (1997), « Pioneering new geographical markets », Journal of Marketing Management, vol. 13, $\mathrm{n}^{\text {os }} 1-3$, p. 89-104.

BourCIEU, S. (2000), «L'internationalisation des PME. Du déterminisme au volontarisme stratégique », Gestion, vol. 25, no 1, p. 12-22.

BOURNOIS, F. et P. LOUART (1998), «L'Europe de l'Est dix ans après », Revue française de gestion, janvier-février, p. 72-79.

BRÉCHET, J.-P. (1996), Gestion stratégique. Le développement du projet d'entreprendre, Paris, ESKA.

CAlori, R. et Y. HARvatopoulos (1988), «Diversification : les règles de conduite », Harvard l'Expansion, printemps, p. 48-59.

CAvusgiL, S.T. (1984), «Differences among exporting firms based on their degree of internationalization », Journal of Business Research, vol. 12, p. 195-208.

CHANDLER, A.D. (1986), « The evolution of modern global competition », dans M.E. Porter, Competition in Global Industries, Boston, Harvard Business School Press.

Czinkota, M.R. et W.J. Johnston (1983), «Exporting: does sales volume make a difference? », Journal of International Business Studies, vol. 14, n 1, p. 147-153.

DEMIL, B. (1998), «Le pionnier et les suiveurs. Les comportements stratégiques face à la réglementation », Revue française de gestion, juin-août, p. 107-116.

DesreumauX, A. (1993), Stratégie, Paris, Précis Dalloz.

DETRIE, J.-P. et al. (1997), STRATEGOR. Stratégie, structure, décision, identité, Paris, Dunod.

DONCKELS, R. et J. LAMBRECHT (1995), «La coentreprise comme lien de collaboration entre les PME des pays développés et PVD», Revue internationale PME, vol. 8 , no 1 , p. 9-29. 
FERnANDEZ, G. et A. NoËL (1994), «PME, mondialisation et stratégies », Revue internationale PME, vol. 6, $\mathrm{n}^{\text {os }} 3-4$, p. 145-163.

Gibiat, M. (1994), «Les modèles intégrés de la décision d'exporter en PME/PMI : synthèse des recherches depuis 20 ans », Revue internationale PME, vol. 7, $\mathrm{n}^{\circ} 2$, p. 11-29.

JULIEN, P.-A. (1994), « Mondialisation des marchés et types de comportements des PMI », Cahier de recherche du GREPME, Université du Québec à Trois-Rivières, $\mathrm{n}^{\text {os }}$ 94-22, $23 \mathrm{p}$.

Julien, P.-A., A. Joyal, L. Deshaies et C. Ramangalahy (1996), « Typologie des comportements stratégiques des PME exportatrices », Gestion, vol. 21, nº 1, p. 29-37.

KHANNA, T. et K. PALEPU (1997), «Why focused strategies may be wrong for emerging markets? », Harvard Business Review, juillet-août, p. 41-51.

Koenig, G. (1996), Management stratégique. Paradoxes, interactions et apprentissages, Paris, Nathan.

LARCON, J.-P. (1996), «Europe de l'Est : une mosaïque d'opportunités », Revue française du marketing, $\mathrm{n}^{\circ} 160$, p. 5-14.

Le Moigne, J.L. (1995), Les épistémologies constructivistes, Paris, Presses universitaires de France, Coll. : «Que sais-je?».

LEVITT, T. (1983), « The globalization of markets », Harvard Business Review, mai-juin, p. $92-102$.

Liouville, J. et C. NANOPOUlos (1998), « Stratégie de spécialisation et compétitivité des PME en environnement global », Gestion 2000, mars-avril, p. 31-50.

Luthans, F., R. PATRick et B. Luthans (1995), « Doing business in Central and Eastern Europe : political, economic and cultural diversity », Business Horizons, septembreoctobre, p. 9-16.

MachKova, H. (1998), « Pratiques et politiques de marketing en Europe de l'Est », Revue française de gestion, janvier-février, p. 107-115.

MARCHESNAY, M. (1992), «Les stratégies de spécialisation », dans J.-P. Helfer et J. Orsoni, Encyclopédie du Management, 2 tomes, Paris, Vuibert, p. 773-779.

MARTINET, A.-C. et al. (1990), Épistémologies et sciences de gestion, Paris, Economica.

MERCIER-SuIssa, C. (1998), «De nouvelles pratiques de management en Russie », Revue française de gestion, janvier-février, p. 80-90.

MINISTÈRE DE L'INDUSTRIE (1993), PMI 93. Les comportements stratégiques des entreprises industrielles de taille moyenne en France et en Allemagne face aux espaces de concurrence européens et mondiaux, Paris, Ministère de 1'Industrie.

OHMAE, K. (1991), Le génie du stratège, Paris, Dunod, Édition originale (1982), The Mind of the Strategist, New York, McGraw-Hill Book Company.

PATUREL, R. (1995), «L'environnement russe est-il favorable à l'entrepreneuriat ? », Document de recherche, CERAG, Université Pierre-Mendès-France, Grenoble, 16 p.

PATUREL, R. (1997), «Les manœuvres stratégiques génériques des entreprises », Économies et Sociétés, Sciences de Gestion, nos 7-8, p. 93-118.

Revue internationale P.M.E., vol. 14, nº 1, 2001

(C) 2001 - Presses de l'Université du Québec

Édifice Le Delta I, 2875, boul. Laurier, bureau 450, Sainte-Foy, Québec G1V 2M2 - Tél. : (418) 657-4399 - www.puq.uquebec.ca 
Paturel, R. et J.L. LeVet (1997), «Comparaison et explication des stratégies des PMI françaises et allemandes », Revue internationale PME, vol. 10, nº 2, p. 81-108.

Plane, J.-M. (1999), «Considérations sur l'approche ethnométhodologique des organisations », Revue française de gestion, mars-mai, p. 44-53.

Pras, B. et A. Boutin (1995), Les Euro-PMI, Paris, Economica.

RichET, X. (1991), «Au défi d'entreprendre. Privatisation, réorganisation industrielle et stratégies d'entreprise », Économie et Humanisme, no 317, p. 19-28.

SAPIR, J. (1995), « Culture économique, culture technologique, culture organisationnelle: les exemples russes et soviétiques », Problèmes économiques, $\mathrm{n}^{\circ}$ 2441, octobre, p. 11-18. Parution initiale dans Cahiers du monde russe, vol. 36, $\mathrm{n}^{\text {os }} 1-2$.

SAPORTA, B. (1993), «Les enjeux de l'ouverture internationale des PME-PMI », CREGE, Travaux de recherches I.9307, $15 \mathrm{p}$.

SAPORTA, B. (1997), «Stratégies des petites et moyennes entreprises », dans P. Joffre et Y. Simon (1997), Encyclopédie de gestion, $2^{2}$ édition, Paris, Economica, p. 3105-3128.

SESSI (1998), L'état des PMI, Édition 1998, Paris, Ministère de l'Économie, des Finances et de l'Industrie.

THÉRIN, F. (1995), «L'implication à l'export des PME/PMI : le cas des sous-traitants de l'industrie aéronautique civile en Midi-Pyrénées », Revue internationale PME, vol. 8, $\mathrm{n}^{\mathrm{o}} 2$, p. 119-146.

THIETART, R.-A. et al. (1999), Méthodes de recherche en management, Paris, Dunod, 535 p.

WELCH, L. S. et R. LUOSTARINEN (1988), «Internationalization : evolution of a concept », Journal of General Management, vol. 14, nº 2, p. 34-64. 